\title{
Evaluation of P Dispersion and P Dispersion Recovery in Patients with Pseudoexfoliative Syndrome
}

\author{
Cosgun Ayhan ${ }^{1}$, Gunes Alper ${ }^{2}$ \\ ${ }^{1}$ Department of Cardiology, Sincan State Hospital, Ankara, Turkey \\ ${ }^{2}$ Department of Ophthalmology, Tokat Gaziosman Pasa University, Faculty of Medicine, Tokat, Turkey \\ *Correspondence author - Cosgun Ayhan; ayhancosgun@gmail.com
}

Received 23 April 2019;

Accepted 18 May 2019;

Published 22 May 2019

\begin{abstract}
Background: Pseudoexfoliative syndrome (PEX) is an old-age disease characterized by the accumulation of fibril materials in both the eye and extraocular organs. PEX is associated with many morbid and mortal cardiovascular diseases. The aim of our study was to investigate the effects of the stress test on P Wave dispersion (PWD) in patients with PEX. Patients and Methods: Forty patients who presented to the ophthalmology outpatient clinic between February 2018 and September 2018 and diagnosed as PEX in the ophthalmologic examination were included in the study. As a control group, forty-four patients, age and gender-matched, who applied to the cardiology outpatient clinic, diagnosed in the outpatient clinic of ophthalmology non-PEX, were included in the study. The study and control group were subjected to treadmill exercise test (TET) according to Bruce protocol. Results: The recovery PWD value of the study group in the third minutes was $34.4 \pm 4.35 \mathrm{~ms}$ and rest PWD value of study group $32.925 \pm 5.17 \mathrm{~ms}$. There was no statistically significant difference between the two values $(\mathrm{t}=1.37992, \mathrm{p}=0.08)$. The basal PWD value of the control group was $31.79 \pm 3.9 \mathrm{~ms}$, the recovery PWD value of the control group in the second minute $32.65 \pm 3.48 \mathrm{~ms}$. There was no statistically significant difference between the two values $(t=1.09495, p=0.13)$. Conclusion: The most interesting result of the study was that the difference between the recovery PWD value and the resting PWD value in the study group was statistically insignificant in the third recovery minute, whereas in the control group it occurred in the second minute.
\end{abstract}

Keywords: Pseudoexfoliative syndrome, $P$ dispersion, $P$ dispersion recovery time, effort test.

\section{Introduction}

PEX is a disease characterized by the accumulation of extracellular fibrillary material in the outside organs of the eye and in the eye. Extraocular accumulation is associated with cardiovascular complications. PEX is associated with myocardial infarction, aortic aneurysm, congestive heart failure, and arrhythmias. ${ }^{[1]}$

Atrial fibrillation (AF) is one of the most common arrhythmias and originates from one or more focal activation centers. Multiple re-entry wavelets from focal activation centers cause atrial fibrillation. ${ }^{[25]} \mathrm{AF}$ causes increased morbidity and hospitalization with or without structural heart disease. ${ }^{[26]}$ As known during the exercise, sympathetic activity increases and parasympathetic activity decreases. Therefore, the majority of arrhythmias and sudden cardiac deaths can occur immediately after severe exercise. $^{[27]}$

PEX is an increasing disease with age (12). AF, despite the increases in many diseases, increases with age even if there is no underlying structural heart disease. ${ }^{[28]}$ PWD is accepted as a risk factor of AF with or without a systemic disease ${ }^{[7]} \mathrm{PWD}$ is defined as the difference between the longest $\mathrm{P}$ wave and the shortest $\mathrm{P}$ wave in the superficial electrocardiogram (ECG). In patients with atrial fibrillation, it is well known that increased P-wave duration and PWD reflect the prolongation of Intra- and interatrial conduction time and non-homogeneous spread of sinus impulses. ${ }^{[29]} \mathrm{P}$-wave duration and PWD have been reported to be influenced by the autonomic tone and intensive exercise. ${ }^{[30]}$

The aim of our study was to investigate the PWD recovery time and PWD values stimulated by TET in PEX patients and the control group.

\section{Materials and Methods}

Study Design: The present study was prospective, cross-sectional, and observational. All procedures performed in studies involving human participants were in accordance with the ethical standards of the Sincan State Hospital Research Committee and with the 1964 Helsinki declaration and its later amendments or comparable ethical standards. All patients were informed of the study protocol and written permission was obtained from each subject. 
Study Population: Forty patients who presented to the ophthalmology outpatient clinic, between February 2018 and September 2018 and diagnosed as PEX in the ophthalmologic examination, were included in the study. There were 28 males and 12 females in the study group (70\% male, $30 \%$ female). The mean age of the study group was $65.79 \pm 4.59$ years. The control group consisted of 44 patients who presented to the cardiology outpatient clinic, had also diagnosed without PEX in the ophthalmology outpatient clinic, between February 2018 and September 2018. The control group consisted of 30 male and 14 female (68.18\% male, $31.82 \%$ female). The mean age of the control group was $65.10 \pm$ 5.36 years. Exclusion criteria were; the patients with previous coronary artery disease and those receiving treatment for any reason, with any systemic disease such as hypertension and diabetes mellitus, impaired liver, renal and thyroid function test, those with electrolyte disturbance in routine blood examination, previously diagnosed with COPD and receiving bronchodilator therapy, having any systemic disease such as diabetes mellitus or hypertension, having impaired liver or kidney function test, having electrolyte defect in routine blood examination, having anemia or thyroid dysfunction, having a disease that may affect the heart, having tension arterial above 140/90 mm $\mathrm{Hg}$, having coronary artery disease before, having positive effort test which indicates significant coronary arterial disease, presence of a cardiac pacemaker, patients with a diagnosis of atrial fibrillation or having AF rhythm in superficial electrocardiograms and patients with orthopedic disorders who were unable to perform TET.

Methods: Patients diagnosed with PEX in the ophthalmology outpatient clinic were included in the study. PEX was diagnosed by biomicroscopy. After taken the routine 12-lead ECG ${ }^{[9]}$ of the study and control group patients, the patients underwent TET according to the Bruce protocol. ${ }^{[10]}$ TET was terminated after $88 \%$ of the target pulse. Resting and values were measured in routine ECG and TET records. The basal and values, the values of the peak level, first, second and third recovery period PWD values were taken.
After the recordings of the patients were taken, they were calculated manually by the same cardiologist with the digital ruler and magnifying glass. P-wave duration was defined as the time measured from the onset to the end of the P-wave deflection. The onset of the P-wave was considered as the junction between the isoelectric line and the first visible upward or downward slope of the trace. The return of the trace to the isoelectric line was considered to be the end of the P-wave. PWD was defined as the difference between the maximum and minimum P-wave duration (Pmax and Pmin, respectively) occurring in any of the 12 leads. ${ }^{[7]}$ All of these evaluations were performed for both the study and the control group.

Ethical Approval: All procedures performed in studies involving human participants were in accordance with the ethical standards of the Sincan State Hospital Research Committee and with the 1964 Helsinki declaration and its later amendments or comparable ethical standards.

Statistical Analysis: SPSS software [Version X; IBM, Armonk, NY, USA] 20.0 was used for the collection and analysis of the data. Measurements were expressed as mean values with \pm standard deviation. Analyzes were performed using the t-test. To compare the values of the different two groups T-Test Calculator for Independent Two Means and to compare the different values of the same group, T-Test Calculator for Depended Means was used. To compare the proportions $\mathrm{Z}$ test calculator for 2 population proportions were used. For correlation, for two values of the same group, the Pearson Correlation Coefficient Calculator was used. For all tests $\mathrm{p}<0.05$, the differences are statistical significance.

\section{Results}

There was no difference between the study and control group in terms of basal clinic findings and there was no statistically significant difference between the socio-demographic characteristics of the patients (Table 1).

Table 1: The socio-demographic characteristics and basal clinic findings of study and control group

\begin{tabular}{|l|l|l|l|l|}
\hline Variables & Study group & Control group & T or Z value & P value \\
\hline Age, years & $65.44 \pm 11.61$ & $62.38 \pm 10.73$ & $\mathrm{~T}=1.30938$ & 0.09 \\
\hline Men, \% & $70 \%$ & $68.18 \%$ & $\mathrm{Z}=0.1812$ & 0.85 \\
\hline Women, \% & $30 \%$ & $31.82 \%$ & $\mathrm{Z}=-0.1812$ & 0.85 \\
\hline BMI, kg/m & $31.26 \pm 4.63$ & $30.58 \pm 3.89$ & $\mathrm{~T}=1.30361$ & 0.09 \\
\hline Basal BP, mm Hg & $131.34 \pm 8.77$ & $128.58 \pm 7.9$ & $\mathrm{~T}=1.60633$ & 0.056 \\
\hline Basal HR, beat/mn & $82.11 \pm 11.7$ & $79.85 \pm 12.7$ & $\mathrm{~T}=1.19045$ & 0.118 \\
\hline LV mass, gram & $177.45 \pm 43.67$ & $173.56 \pm 41.68$ & $\mathrm{~T}=0.436$ & 0.33 \\
\hline Total cholesterol, mg/dL & $196.74 \pm 35.79$ & $189.48 \pm 40.05$ & $\mathrm{~T}=0.849$ & 0.19 \\
\hline LDL, mg/dL & $133.58 \pm 35.67$ & $141.73 \pm 41.63$ & $\mathrm{~T}=-0.9570$ & 0.17 \\
\hline Triglycerides, mg/dL & $132.67 \pm 24.79$ & $124.58 \pm 25.73$ & $\mathrm{~T}=1.4928$ & 0.069 \\
\hline Hemoglobin, gr/dL & $14.74 \pm 2.6$ & $14.37 \pm 2.8$ & $\mathrm{~T}=0.056$ & 0.47 \\
\hline Calcium, mg/dL & $9.6 \pm 1.1$ & $9.2 \pm 0.94$ & $\mathrm{~T}=0.084$ & 0.46 \\
\hline Sodium, mEq/L & $139.84 \pm 3.8$ & $140.06 \pm 3.1$ & $\mathrm{~T}=-1.525$ & 0.065 \\
\hline
\end{tabular}

Abbr: BMI; Body Mass Index, BP; Blood Pressure, LV; Left Ventricle, LDL; Low-Density Lipoprotein

There was no statistically significant difference between basal heart rates of study and control group. In addition, there was no statistically significant difference between the basal PWD values of the study group and the control group. There was a statistically significant difference between peak, first, second and third recovery PWD values of the study group and control group (Table 2). 
Table 2: The comparison the values of TET in study and control group

\begin{tabular}{|l|l|l|l|l|}
\hline Variables & Study group & Control group & T value & P value \\
\hline Basal HR, beat/mn & $82.11 \pm 11.7$ & $79.85 \pm 12.7$ & $\mathrm{~T}=1.19045$ & 0.118 \\
\hline Basal PWD, ms & $32.92 \pm 5.17$ & $31.79 \pm 3.9$ & $\mathrm{~T}=1.13623$ & 0.12 \\
\hline Peak PWD, ms & $37.92 \pm 5.83$ & $34.5 \pm 3.78$ & $\mathrm{~T}=3.3688$ & $<0.01$ \\
\hline $\mathbf{1}$ min rec. PWD, ms & $36.22 \pm 5.3$ & $33.38 \pm 3.6$ & $\mathrm{~T}=3.3669$ & $<0.01$ \\
\hline $\mathbf{2}$ min rec. PWD, ms & $35.3 \pm 4.87$ & $32.65 \pm 3.48$ & $\mathrm{~T}=3.9108$ & $<0.01$ \\
\hline $\mathbf{3}$ min rec. PWD, ms & $34.4 \pm 4.35$ & $31.90 \pm 3.38$ & $\mathrm{~T}=3.9006$ & $<0.01$ \\
\hline
\end{tabular}

Abbr: HR; Heart Rate, PWD; P Wave dispersion, 1 min rec; First-minute recovery, 2 min rec; Second-minute recovery, 3 min rec; Third-minute recovery

In the study group, there was a statistically significant difference between the resting PWD and peak level PWD, and between peak level PWD and first-minute PWD, between first minute PWD and second-minute recovery PWD and between the second minute PWD and third-minute PWD. In addition, there was a statistically significant difference between basal PWD and first recovery minute PWD, between basal PWD and second-minute recovery PWD. However, there was no statistically significant difference between the basal PWD and the third-minute recovery PWD. This means that the difference between resting PWD and the PWD values of recovery periods became meaningless at the third recovery stage of TET (Table 3 ).

Table 3: The comparison of the values of TET in the study group

\begin{tabular}{|c|c|c|c|}
\hline Variable 1 & Variable2 & T value & $P$ value \\
\hline BPWD, $32.92 \pm 5.17 \mathrm{~ms}$ & PPWD, $37.92 \pm 5.83 \mathrm{~ms}$ & 8.779242 & $<0.01$ \\
\hline PPWD, $37.92 \pm 5.83 \mathrm{~ms}$ & FMRPWD, $36.22 \pm 5.3 \mathrm{~ms}$ & 6.910751 & $<0.01$ \\
\hline FMRPWD, 36.22 $\pm 5.3 \mathrm{~ms}$ & SMRPWD, $35.3 \pm 4.87 \mathrm{~ms}$ & 5.867163 & $<0.01$ \\
\hline SMRPWD, $35.3 \pm 4.87 \mathrm{~ms}$ & TMRPWD, $34.4 \pm 4.35 \mathrm{~ms}$ & 4.388872 & $<0.01$ \\
\hline BPWD, $32.92 \pm 5.17 \mathrm{~ms}$ & FMRPWD, $36.22 \pm 5.3 \mathrm{~ms}$ & 2.8177 & $<0.01$ \\
\hline BPWD, $32.92 \pm 5.17 \mathrm{~ms}$ & SMRPWD, $35.3 \pm 4.87 \mathrm{~ms}$ & 2.1139 & $=0.018$ \\
\hline BPWD, $32.92 \pm 5.17 \mathrm{~ms}$ & TMRPWD, $34.4 \pm 4.35 \mathrm{~ms}$ & 1.37992 & $=0.085$ \\
\hline
\end{tabular}

Abbr: BPWD; Basal P wave dispersion, PPWD; Peak level P Wave dispersion, FMRPWD; First-minute recovery P wave dispersion, SMRPWD; Second-minute recovery $\mathrm{P}$ wave dispersion, TMRPWD; Third-minute recovery $\mathrm{P}$ Wave dispersion

In the control group, there was a statistically significant difference between the basal PWD and peak level PWD, between peak level PWD and first-minute recovery PWD, between first-minute recovery PWD and second-minute PWD. However, there was no statistically significant difference between the second-minute recovery and the third-minute recovery PWD. In addition, there was no statistically significant difference between the basal PWD and the second-minute recovery PWD, between basal PWD and the third-minute recovery PWD. This means that the difference between the basal PWD and the PWds of recovery periods became meaningless in the second stage of TET. This meaningless difference of the control group became earlier one stage then the study group (Table 4).

Table 4: The comparison of the values of TET in the control group

\begin{tabular}{|l|l|l|l|}
\hline Variable $\mathbf{1}$ & Variable2 & T value & P value \\
\hline BPWD, 31.79 $\pm \mathbf{3 . 9} \mathbf{~ m s}$ & PPWD, 34.5 $\pm 3.78 \mathrm{~ms}$ & 3.29825 & $<0.01$ \\
\hline PPWD, 34.5 $\pm \mathbf{3 . 7 8} \mathbf{~ m s}$ & FMRPWD, 33.38 $\pm 3.6 \mathrm{~ms}$ & 1.70579 & 0.05 \\
\hline FMRPWD, 33.38 $\pm \mathbf{3 . 6} \mathbf{~ m s}$ & SMRPWD, 32.65 $\pm 3.48 \mathrm{~ms}$ & 1.68579 & 0.05 \\
\hline SMRPWD, 32.65 $\pm \mathbf{3 . 4 8} \mathbf{~ m s}$ & TMRPWD, 31.90 $\pm 3.38 \mathrm{~ms}$ & 1.49071 & 0.07 \\
\hline BPWD, 31.79 $\pm \mathbf{3 . 9} \mathbf{~ m s}$ & FMRPWD, 33.38 $\pm 3.6 \mathrm{~ms}$ & 1.98641 & 0.02 \\
\hline BPWD, 31.79 $\pm \mathbf{3 . 9} \mathbf{~ m s}$ & SMRPWD, 32.65 $\pm 3.48 \mathrm{~ms}$ & 1.09495 & 0.13 \\
\hline BPWD, 31.79 $\pm \mathbf{3 . 9} \mathbf{~ m s}$ & TMRPWD, $31.90 \pm 3.38 \mathrm{~ms}$ & 0.14583 & 0.44 \\
\hline
\end{tabular}

Abbr: BPWD; Basal P wave dispersion, PPWD; Peak level P Wave dispersion, FMRPWD; First-minute recovery P wave dispersion, SMRPWD; Second-minute recovery $P$ wave dispersion, TMRPWD; Third-minute recovery $P$ Wave dispersion

There was a moderate positive correlation between age and basal PWD values in the study group $(r=0.7117$ and $\mathrm{p}<0.00001)$ (Figure 1). There was also a moderate positive correlation between age and basal PWD values in the control group $(r=0.5129$ and $\mathrm{p}<0.00001)$ (Figure 2). 


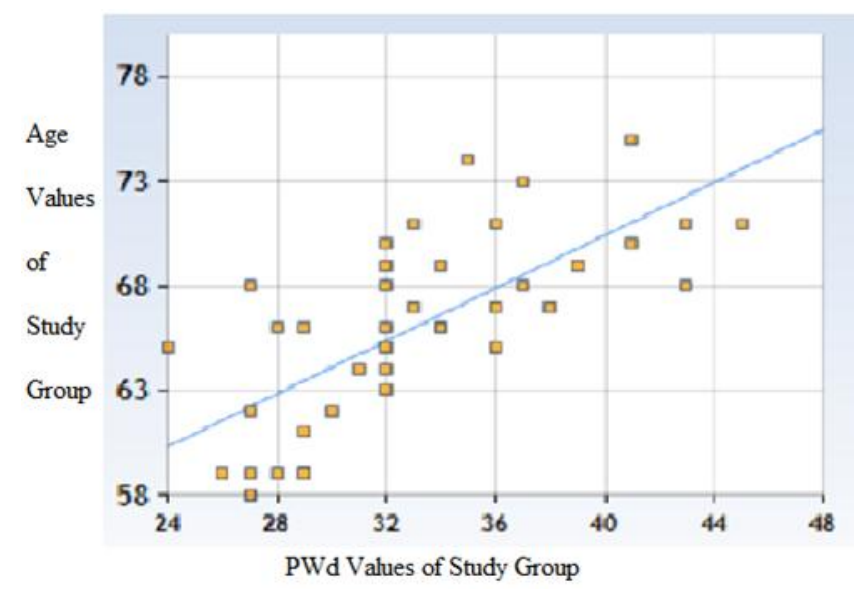

Abbr: PWD; P Wave Dispersion $\mathrm{r}=0.7117$ and $\mathrm{p}<0.00001$

Figure 1: Linear Regression Graphic of Basal PWD and Age of Study Group

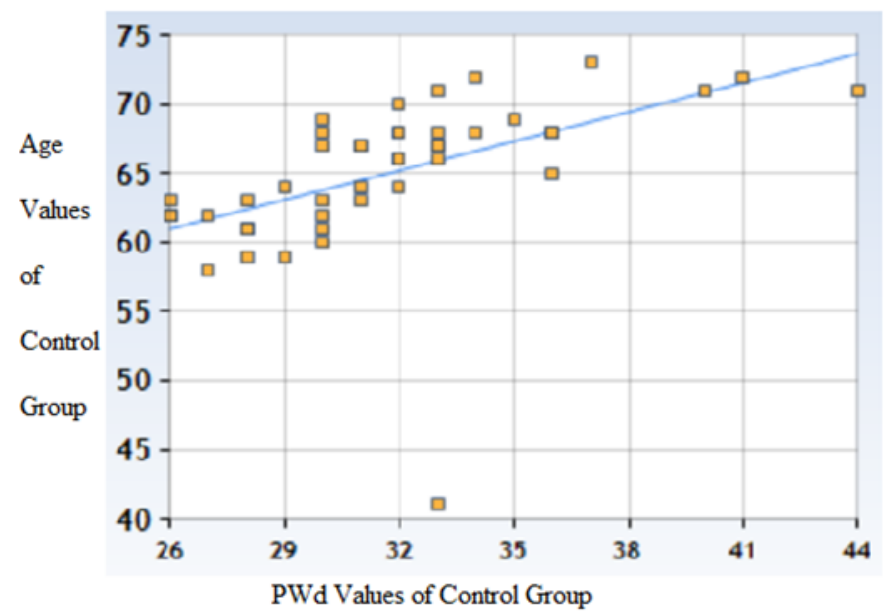

Abbr: PWD; P Wave Dispersion

$\mathrm{r}=0.5129$ and $\mathrm{p}<0.00001$

Figure 2: Linear Regression Graphic of Basal PWD and Age of Control Group

\section{Discussion}

In our study, there were three important results. The first was that peak PWD in the study group is significantly higher than the basal PWD value. This means that the risk of AF with exertion is higher in PEX patients than the rest. The second important result is that the peak exercise PWD value in the study group was statistically higher than the peak exercise PWD in the control group. The third result was that while the difference between the basal PWD value and the recovery PWD values, as a result of the stress test, became statistically insignificant in the study group at the third recovery minute. In the control group, this difference became meaningless in the second recovery minute.

This result showed that the vagal autonomic system worked less in PEX patients than the control group. The reduction in Heart Rate Recovery (HRR) indicates a decrease in vagal autonomic function. ${ }^{[11]}$ However, PWD recovery has not been studied until now.
PEX is directly proportional to age. ${ }^{[12]}$ In addition to this, it causes increased cardiovascular risk factors. PEX is associated with myocardial infarction, aortic aneurysm, congestive heart failure, and arrhythmias. ${ }^{[1]}$ In a review, 18 studies on PEX were examined and PEX was found to be associated with ischemic heart disease, aortic aneurysm, and homocystinuria, but was not correlated with angina pectoris, acute myocardial infarction, hypertension, and chronic ischemic heart disease. ${ }^{[5]}$ It was also found that the chance of arrhythmia increased in PEX without coronary artery disease. ${ }^{[6]}$

Atrial fibrillation is one of the leading causes of heart failure and CVE worldwide. ${ }^{[18,19]}$ It also brings significant financial cost burdens. ${ }^{[20]}$ The incidence of $\mathrm{AF}$ is greater than 15 percent in patients over 85 years of age. ${ }^{[21]}$ Although a significant relationship was found between AF and obesity, diabetes mellitus and metabolic syndrome, the relationship between PEX and AF was not investigated. ${ }^{[22-24]}$ This study was perhaps the first study to investigate the relationship between PEX and AF risk.

AF has many complications. Especially the loss of atrial contribution and the tendency to thromboembolism change the course of the disease. The most important morbid and mortal event is the cerebrovascular event (CVE). ${ }^{[13]}$ Atrial fibrillation is more common with age such as PEX. ${ }^{[14]} \mathrm{PWD}$ is adopted as a risk factor for $\mathrm{AF}$ with or without a systemic disease. ${ }^{[7]}$ In one study, $\mathrm{P}$ duration, PWD and recovery time were found to be longer in the patients with paroxysmal atrial fibrillation than with the control group. ${ }^{[15]}$

PWD has been investigated in many diseases and found to be closely related to AF. Some of them have been investigated in patients with non-structural heart disease, hypertension, coronary artery disease, in the patients undergoing bypass surgery, patients with congenital heart disease, in patients with cardiac or noncardiac symptoms, and non-cardiac diseases. ${ }^{[16]}$ Although Dogan U. et al., ${ }^{[17]}$ found a very close relationship between proximal AF and $\mathrm{P}$ duration, they proved that $\mathrm{P}$ duration was no independent risk factor. It was also found that PWD was closely associated with paroxysmal AF in stroke patients. In the meantime, PWD was determined as an independent risk factor for paroxysmal atrial fibrillation in logistic regression analysis. So we looked at the PWD values in our study.

In one study, it was stated that sympathetic activity increased with exercise test and vagal activity was at the recovery phase after the exercise test. In this study, the HRR time was examined at the end of the exercise test, but PWD recovery time was not observed. ${ }^{[16]}$

In our study, there was no statistically significant difference between the control group and resting PWD values in patients with PEX. In addition, peak PWD was significantly higher in the study group than both the control group and the basal PWD value of the study group. Besides, the difference between the basal PWD value and the PWD recovery values in the study group was statistically insignificant at the third recovery minute, while this occurred at the second recovery minute in the control group.

\section{Conclusion}

In conclusion, in PEX patients we found that we were experiencing an indirect reduced vagal activity and this might be a predictor of future cardiovascular events. In addition, we suggest that patients with PEX should not make intensive exercise. 


\section{Conflict of Interest}

The authors declare that they have no conflict of interest.

\section{Study Limitations}

In our study, the number of cases is little, healthier data can be made with larger case studies. And also, we calculated PWD values as manually by a digital caliper. And we couldn't use 24 hours Holter monitorization to determine AF in patients with PEX. In addition, long-term studies should be made in order to observe the effect of medical therapy on the PWD and AF induced by the exercise test in patients with PEX. More comprehensive and longterm follow-up studies should be performed to obtain healthier data.

\section{References}

[1] Gonen KA, Gonen T, Gumus B. Reply: Pseudoexfoliation syndrome and cardiovascular disease: studies must control for all cardiovascular risk factors. Eye 2013;27:1329. https://doi.org/10.1038/eye.2013.185. [Pubmed]

[2] Yusuf IH. Pseudoexfoliation syndrome and cardiovascular disease: studies must control for all cardiovascular risk factors, Eye (Lond). 2013 Nov; 27(11):

1328-1329. https://doi.org/10.1038/eye.2013.184. [Pubmed]

[3] Gonen KA, Gonen T, Gumus B. Renal artery stenosis and abdominal aorta aneurysm in patients with pseudoexfoliation syndrome, Eye (Lond). 2013 Jun; 27(6):735-41. https://doi.org/10.1038/eye.2013.56. [Pubmed]

[4] Hietanen J, Soisalon-Soininen S, Kivelä T, at all. Evaluation of the clinical association between exfoliation syndrome and abdominal aortic aneurysm. Acta Ophthalmol Scand 2002 Dec;80(6):617-9.PMID: 12485282. [Pubmed]

[5] Siordia JA, Franco J, Golden TR, at all. Ocular Pseudoexfoliation Syndrome Linkage to Cardiovascular Disease. Curr Cardiol Rep. 2016 Jul;18(7):61. https://doi.org/10.1007/s11886-016-0738-5. [Pubmed]

[6] Brajkovic J, Surac IK, Ercegovic A, at all. Ocular Pseudoexfoliation syndrome and internal systemic diseases. Acta Clin Croat. 2007; 46(suppl 1):5761.PMID: 9588401. [Pubmed]

[7] Dilaveris PE, Gialafos EJ, Sideris SK. Simple electrocardiographic markers for the prediction of paroxysmal idiopathic atrial fibrillation. Am Heart J 1998; 135 (5): 733-8.PMID: 9588401. [Pubmed]

[8] Demir AK, Demir S, Arısoy A, at all. The Association of Pseudoexfoliation Syndrome with Ventricular Repolarization Dynamics. Asian Journal of Medicine and Health. 5(2): 1-7, 2017; Article no.AJMAH.34397.

[9] $10 \mathrm{mV}, 25 \mathrm{~mm} / \mathrm{sn}, 12$ derivations, Clinical Guidelines by Consensus, Recording a standard 12-lead electrocardiogram, an approved methodology by the Society for Cardiological Science \& Technology SCST.

[10] Bruce RA., Exercise testing of patients with coronary heart disease. Principles and normal standards for evaluation. Ann Clin Res. 1971 Dec;3(6):323-32.PMID: PMID: 5156892. [Pubmed]
[11] Jae SY, Bunsawat K, Kunutsor SK, at all. Relation of Exercise Heart Rate Recovery to Predict Cardiometabolic Syndrome in Men. Am J Cardiol. 2018 Nov 24. pii: S0002-9149(18)32098-8. https://doi.org/10.1016/j.amjcard.2018.11.017. [Pubmed]

[12] Kling F, Colin J. Potential association of pseudoexfoliation syndrome (PEX) with age-related macular degeneration (ARMD). J Fr Ophthalmol. 2001 Jan;24(1):7-12. PMID: 11240465. [Pubmed]

[13] Hahne K, Mönnig G, Samol A. Atrial fibrillation and silent stroke: links, risks, and challenges. Vasc Health Risk Manag. $2016 \quad$ Mar 7;12:65-74. https://doi.org/10.2147/VHRM.S81807. [Pubmed]

[14] Charlemagne A, Blacher J, Cohen A, at all. Epidemiology of atrial fibrillation in France: extrapolation of international epidemiological data to France and analysis of French hospitalization data. Arch Cardiovasc Dis. $2011 \quad$ Feb;104(2):115-24. https://doi.org/10.1016/j.acvd.2010.11.012. [Pubmed]

[15] Yigit Z, Akdur H, Ersanli M, at all. The effect of exercise to $\mathrm{P}$ wave dispersion and its evaluation as a predictor of atrial fibrillation. Ann Noninvasive Electrocardiol. 2003 Oct;8(4):308-12. PMID: 14516287. [Pubmed]

[16] Okutucu S, Aytemir K, Oto A. P-wave dispersion: What we know till now? JRSM Cardiovasc Dis. 2016 Mar 21;5:2048004016639443. https://doi.org/10.1177/2048004016639443. [Pubmed]

[17] Dogan U, Dogan EA, Tekinalp M, at all. P-wave Dispersion for Predicting Paroxysmal Atrial Fibrillation in Acute Ischemic Stroke. Int J Med Sci. 2012; 9(1): 108-114. PMID: 22211098. [Pubmed]

[18] Krahn AD, Manfreda J, Tate RB. The natural history of atrial fibrillation: Incidence, risk factors, and prognosis in the Manitoba follow-up study. Am J Med 1995; 98:476-84. $\quad$ https://doi.org/10.1016/S0002-9343 (99)80348-9. [Pubmed]

[19] Vidaillet H, Granada JF, Chyou P. A population-based study of mortality among patients with atrial fibrillation or flutter. Am J Med 2002; 113:365-70. PMID: 12401530. [Pubmed]

[20] Leyden JM, Kleinig TJ, Newbury J. Adelaide. stroke incidence study: declining stroke rates but many preventable cardioembolic strokes. Stroke 2013; 44:1226-31.

https://doi.org/10.1161/STROKEAHA.113.675140. [Pubmed]

[21] Lowres N, Neubeck L, Redfern J. Screening to identify unknown atrial fibrillation. A systematic review. Thromb Haemost 2013; 110:213-22. https://doi.org/10.1160/TH13-02-0165. [Pubmed]

[22] Watanabe H, Tanabe N, Watanabe T. Metabolic syndrome and risk of development of atrial fibrillation. Circulation 2008; 117:1255-60. https://doi.org/ 10.1161/CIRCULATIONAHA.107.744466. [Pubmed]

[23] Ball J, Thompson DR, Ski CF. Estimating the current and future prevalence of atrial fibrillation in the Australian adult population. Med J Aust 2015; 202:32-5. PMID: 25588442. [Pubmed]

[24] Dagres N, Anastasiou-Nana M. Atrial Fibrillation and Obesity. J Am Coll Cardiol 2010; 55:2328-9. https://doi.org/ 10.1016/j.jacc.2010.01.045. [Pubmed] 
[25] Camm AJ, Kirchhof P, Lip GY, et al. Guidelines for the management of atrial fibrillation: the Task Force for the Management of Atrial Fibrillation of the European Society of Cardiology (ESC). Europace 2010; 12: 13601420. https://doi.org/ 10.1093/europace/euq350 [PubMed]

[26] Lodder J, Bamford JM, Sandercock PA, at all. Are hypertension or cardiac embolism likely causes of lacunar infarction? Stroke. 1990; 21:375381.PMID:2309260. [Pubmed]

[27] San Antonio R, Guasch E, Tolosana JM, at all. Determining the best approach to reduce the impact of exercise-induced atrial fibrillation: prevention, screening, or symptom-based treatment? Expert Rev Cardiovasc Ther. $2018 \quad$ Nov $19 . \quad$ https://doi.org/ 10.1080/14779072.2019.1550720. [Pubmed]

[28] Justus M. Anumonwo, José Jalife, and Daniel R. Goldstein. Triple threat: adiposity, aging, atrial fibrillation. Aging (Albany NY). 2017 Nov; 9(11): 22352236. https://doi.org/ 10.18632/aging.101318. [Pubmed]

[29] Aytemir K, Ozer N, Atalar E, et al. P wave dispersion on 12-lead electrocardiography in patients with paroxysmal atrial fibrillation. Pacing Clin Electrophysiol 2000; 23: 1109-1112. PMID: 10914366 [PubMed]

[30] Gunes Y, Tuncer M, Guntekin U. Lack of diurnal variation of P-wave and QT dispersions in patients with heart failure. Pacing Clin Electrophysiol. 2008; 31:974978. https://doi.org/10.1111/j.1540-8159.2008.01124. [PubMed].
Abbreviations
AF: Atrial Fibrillation
BHR: Basal Heart Rate
BPWD: Basal P Wave dispersion
CVE: Cerebro Vascular Events
ECG: Electro Cardio Graphy
FMRPWD: First Minute Recovery of P Wave dispersion
PEX: Pseudoexfoliative sydrome
PPWD: P Wave dispersion of peak level of treadmill exercise test PWD: P wave dispersion
SMRPWD: Second Minute Recovery P Wave dispersion TET: Treadmill Exercise Test
TMRPWD: Third Minute Recovery P Wave dispersion
TTE: Transthoracic Echocardiography 